\title{
Evolución de mortalidad y estado actual de una población infectada por VIH controlada en un centro multiprofesional
}

\author{
Marcelo Wolff $\mathbf{R}^{\mathbf{1}, \mathbf{2}}$, Patricia Alvarez $\mathbf{P}^{\mathbf{1} a}$, Ingrid Flores $\mathbf{S}^{\mathbf{1}}$, \\ Rebeca Northland $A^{1,2}$, Claudia Wolff $L^{b}$. \\ Evolution of mortality and current \\ status of an HIV-infected population \\ cared for at a comprehensive \\ HIV/AIDS center in Chile
}

Background: Chile, a middle-income country with an HIV epidemic of moderate proportions (global infection rate $0.2 \%$ ) began a government sponsored, free, highly active antiretroviral therapy (HAART) for patients from the public health system in 2001 reaching in 2004 a 100\% coverage. Arriaran Foundation (AF) is the largest public AIDS care center for adults in the country. Aim: To show the present status of the AF population and the evolution of mortality. Material and Methods: Review of AF database from 1991-2004 that at 12/31/2004 had a total cumulative population of 2,259 adult patients; an active census of 1,065 patients and admitting rate 160-190 patients per years. Results: The global mortality registered was $33.4 \%$, with decreasing annual mortality from $15.7 \%$ of its active population in 1995 to $1.9 \%$ in 2004 . As of 12/31/2004, 817 patients (76.7\%) were receiving antiretroviral therapy (ART); and $19.3 \%$ either did not require nor accept it. Thirty one percent received Combivir $^{\circledR}$ and nevirapine, with undetectable viral load ( $<400$ copies per $\mathrm{ml}$ ) in $78 \%$. Thirty percent received Combivir ${ }^{\circledR}$ and efavirenz with undetectable viral load in $80 \%$ at last count. Both regimens were used mainly as first therapy. Lopinavir/ritonavir was received by $6.3 \%$ of patients, mainly for post failure therapy and $58 \%$ had undetectable viral load. A baseline CD4 count $<200 \mathrm{x} \mathrm{mm}^{3}$ was present in $70 \%$ of patients, $45.3 \%$ had a count below 100 and $47.8 \%$ had clinical AIDS. At the last follow up assessment, CD4 count was $<200$ in $36.8 \%,<100$ in $10.6 \%$ and $200-350$ in $44.9 \%$. Conclusion: The expanded access program to ART in a public, comprehensive AIDS care center in Chile has been highly successful in reaching high undetectability (75\%), reducing mortality and improving immune status despite very advanced baseline disease (Rev Méd Chile 2006; 134: 581-8).

(Key words: Acquired immunodeficiency syndrome; Antiretroviral therapy, highly active; HIV seropositivity)

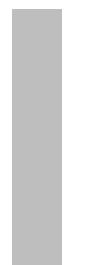

Recibido el 7 de junio, 2005. Aceptado el 26 de octubre, 2005.

Trabajo presentado en forma parcial y resumida en la $12^{\text {th }}$ Conference on Retrovirus and Opportunistic Infections (CROI), Febrero 22-25, 2005; Boston, Estados Unidos de Norteamérica (EE.UU), (resumen $\mathrm{n}$ o 624).

${ }^{1}$ Fundación Arriarán, Hospital Clínico San Borja Arriarán; ${ }^{2}$ Facultad de Medicina Universidad de Chile.

aEnfermera universitaria

bInterna de la carrera de medicina

Correspondencia a: Marcelo Wolff R. 23 de febrero 8630-N,

Santiago, Chile. E mail: mwolff@vtr.net 
$\mathrm{L}$ a pandemia del síndrome de inmunodeficiencia adquirida (SIDA), principal problema de salud pública mundial actual, ha causado la muerte de más de 20 millones de personas desde su aparición, 3,1 millones sólo en el último año ${ }^{1}$. El desarrollo de la terapia antirretroviral (TAR) moderna (combinación de al menos 3 drogas antirretrovirales), en 1996, supresiva de la replicación viral pero no curativa en términos de erradicación del agente, ha tenido un impacto espectacular en la marcada reducción en morbimortalidad relacionada a la epidemia ${ }^{2-4}$. El acceso amplio a TAR ya es práctica habitual en países industrializados y en algunos de recursos medios. Chile es uno de estos últimos y, desde 2001, cuenta con un programa de acceso expandido a TAR para la población infectada que se atiende en el sistema público de salud (85\% de los infectados bajo control de salud en el país), con cobertura actual de $100 \%$. Desde julio de 2005, esta terapia está también garantizada para la población atendida por otros prestadores de salud, en base a la reforma sanitaria y el plan AUGE (Acceso Universal con Garantías Explícitas) ${ }^{5}$. Localmente, la reducción de morbimortalidad ha sido también muy importante, tanto en centros individuales como a nivel global nacional ${ }^{6-8}$. Fundación Arriarán (FA): Centro de Atención Integral de Enfermedad por VIH es una organización no gubernamental, sin fines de lucro, conformada por un equipo multiprofesional que funciona en dependencias del Hospital San Borja Arriarán, en el sector central de Santiago. Cuenta con médicos infectólogos, enfermeras, asistentes sociales, psicólogos, odontólogo y personal técnico-administrativo propio. Por medio de un convenio de colaboración mutua con el Servicio de Salud Metropolitano Central, se encarga de ejecutar el componente asistencial ambulatorio del programa VIH/SIDA de este servicio. FA atiende al mayor número de pacientes a nivel nacional y su modelo, tanto de infraestructura como de recurso humano, ha sido reproducido en gran medida en la propuesta del plan AUGE para los centros de atención del sistema público de salud ${ }^{9}$.

El objetivo central de este trabajo es mostrar la experiencia de un centro de atención multiprofesional de enfermedad por VIH, desde su creación en 1991 hasta 2004, en términos de:
- Evolución de la mortalidad de la población atendida desde la era pre-terapia antirretroviral a la actualidad.

- El estado de la población atendida en este centro al 31/12/2004 respecto a cobertura de TAR y esquemas terapéuticos utilizados.

- Caracterización del estado de supresión virológica en un corte al 31/12/2004 y resultados inmunológicos respecto al basal previo al inicio de TAR.

\section{MÉTodos}

Estudio descriptivo no experimental y en el que la intervención fue la atención asistencial habitual de la población bajo control, que en un comienzo sólo correspondió a manejo de complicaciones y luego se fue agregando terapia antirretroviral específica con diversos grados de complejidad y efectividad. Se procedió al análisis de los registros de FA y, para la determinación de los resultados, se cerró el censo poblacional al 31 de diciembre de 2004. Se consignó el recuento de CD $4 \mathrm{x} \mathrm{mm}^{3}$ basal (preTAR) de los pacientes en terapia, determinándose la mediana y su distribución porcentual en categorías $>500$, 350-499, 200-349, 100-199 y $<100$, por ser éstos los rangos que determinan pronóstico, medidas preventivas de complicaciones, necesidad de TAR e incluso, resultados de ésta. Se consignó, a través de un corte transversal a tal fecha, los regímenes terapéuticos en uso y, con la misma metodología, a caracterizar el último recuento de CD4 y carga viral (CV) como porcentaje de la población con carga viral $\leq 400$ copias $\mathrm{x}$ $\mathrm{mm}^{3}$ (indetectable) versus $>400$, en pacientes con $\geq 3$ meses de terapia antirretroviral (tiempo necesario para observar algún efecto medible en los parámetros en estudio). No se muestran las características clínicas basales ni al momento del corte en los pacientes. Los resultados de respuesta virológica e inmunológica sólo se determinaron en los pacientes en TAR.

La mortalidad anual fue calculada como porcentaje del total de pacientes (con y sin TAR) atendidos anualmente con fallecimiento documentado durante el año calendario y se obtuvo de los registros institucionales anuales. Los datos incorporados son poblacionales y no individuales. Se aplicó el método de chi cuadrado para determi- 
nar diferencia estadística entre los parámetros comparados basalmente y al 31/12/2004.

\section{RESULTADOS}

Desde marzo de 1991 a diciembre de 2004, FA había atendido a 2.259 pacientes (87\% hombres); al 31/12/ 2004, su población activa era de 1.065 pacientes. En los últimos 3 años los ingresos anuales han variado entre 160 y 190 pacientes. La Figura 1 muestra la evolución de ingresos y fallecimientos anuales de 1991 a 2004.

Mortalidad. La mortalidad global acumulada fue de 33,5\% (756/2.259), la mortalidad anual se muestra en la Figura 2 y ha disminuido progresivamente, de $14 \%$ en 1991, con máximo de 15,7\% en 1995, a 1,9\% en 2004. En la Figura se muestra, además, el tipo de TAR predominante, proporcionada por el programa estatal durante distintos períodos del seguimiento. La Tabla 1 muestra que 817 pacientes $(76,7 \%$ del total) recibían TAR, $42(5 \%)$ estaban en proceso de iniciarla o cambiarla y $209(18,3 \%)$ no la requerían o, si estaba indicada, no deseaban recibirla. Se muestra, también, algunas características de la población.

Regímen es terapéuticos en uso y respuesta virológica. La Tabla 2 muestra los regímenes terapéuticos más frecuentes al momento del corte y el porcentaje de pacientes con CV indetectable. Zidovudina más lamivudina $\left(\right.$ Combivir $\left.^{\circledR}\right)$ era utilizado en $63,4 \%$ de los pacientes como combinación de base y efavirenz y nevirapina como tercera droga en $38,4 \%$ y $33,9 \%$, con indetectabilidad viral en $80 \%$ y $78 \%$, respectivamente. La indetectabilidad de la CV por régimen variaba de $80 \%$ a $44 \%$, y la global fue de $75 \%$. No hubo variación estadísticamente significativa en indetectabilidad de hombres $(73 \%)$ y mujeres $(75 \%)$, ni entre previamente vírgenes a terapia (76\%) y previamente tratados (71\%) (Tabla 3).

Respuesta inmunológica. La Tabla 4 muestra el recuento basal de CD4 en 815 pacientes (99\%). La mediana fue de $129,5 \mathrm{x} \mathrm{mm}^{3} \mathrm{y}$ el porcentaje con CD4 $<200 \mathrm{x} \mathrm{mm}^{3}$, de $70,3 \%(45,3 \%<100)$.

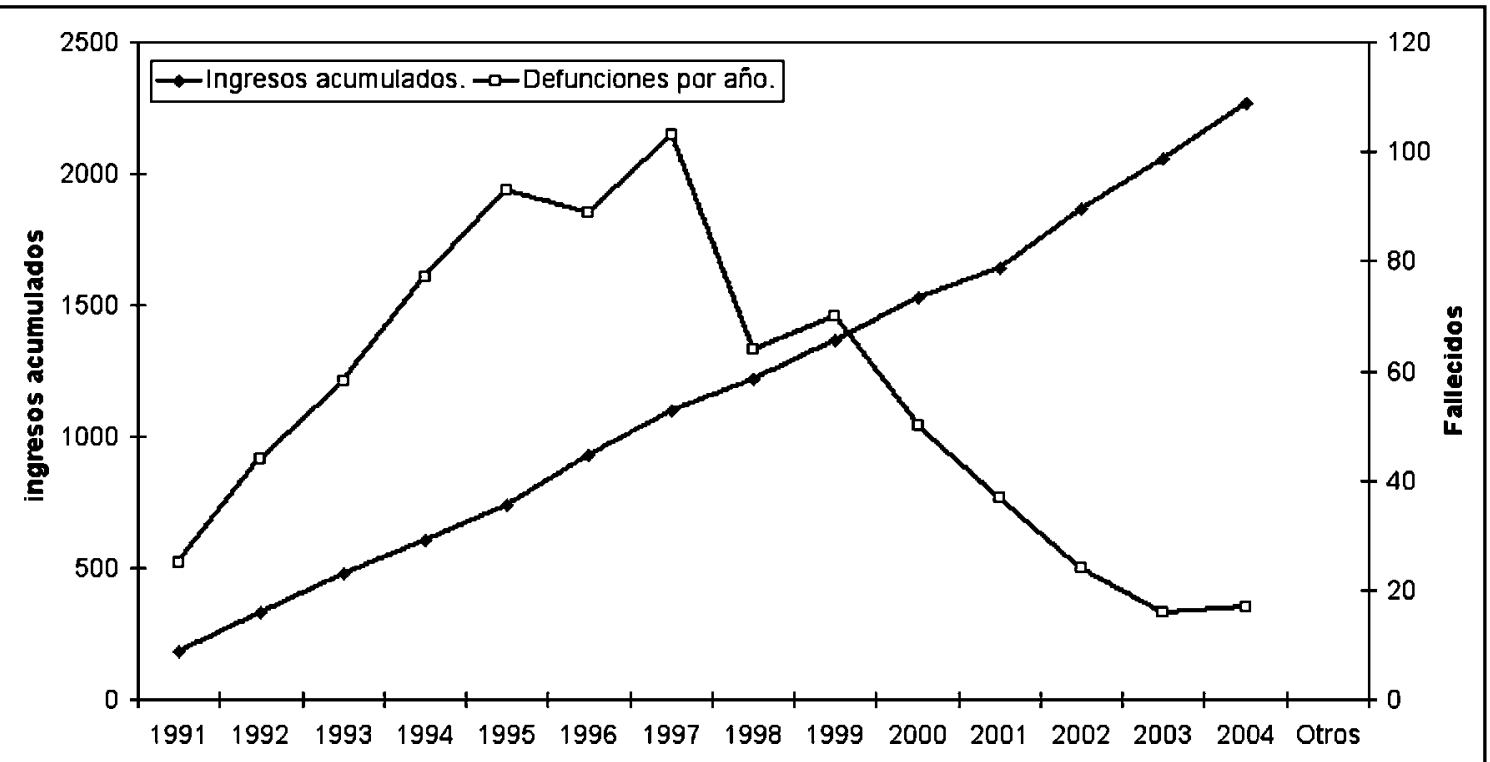

Figura 1. Evolución de ingresos y defunciones. Fundación Arriarán, 1991-2004 


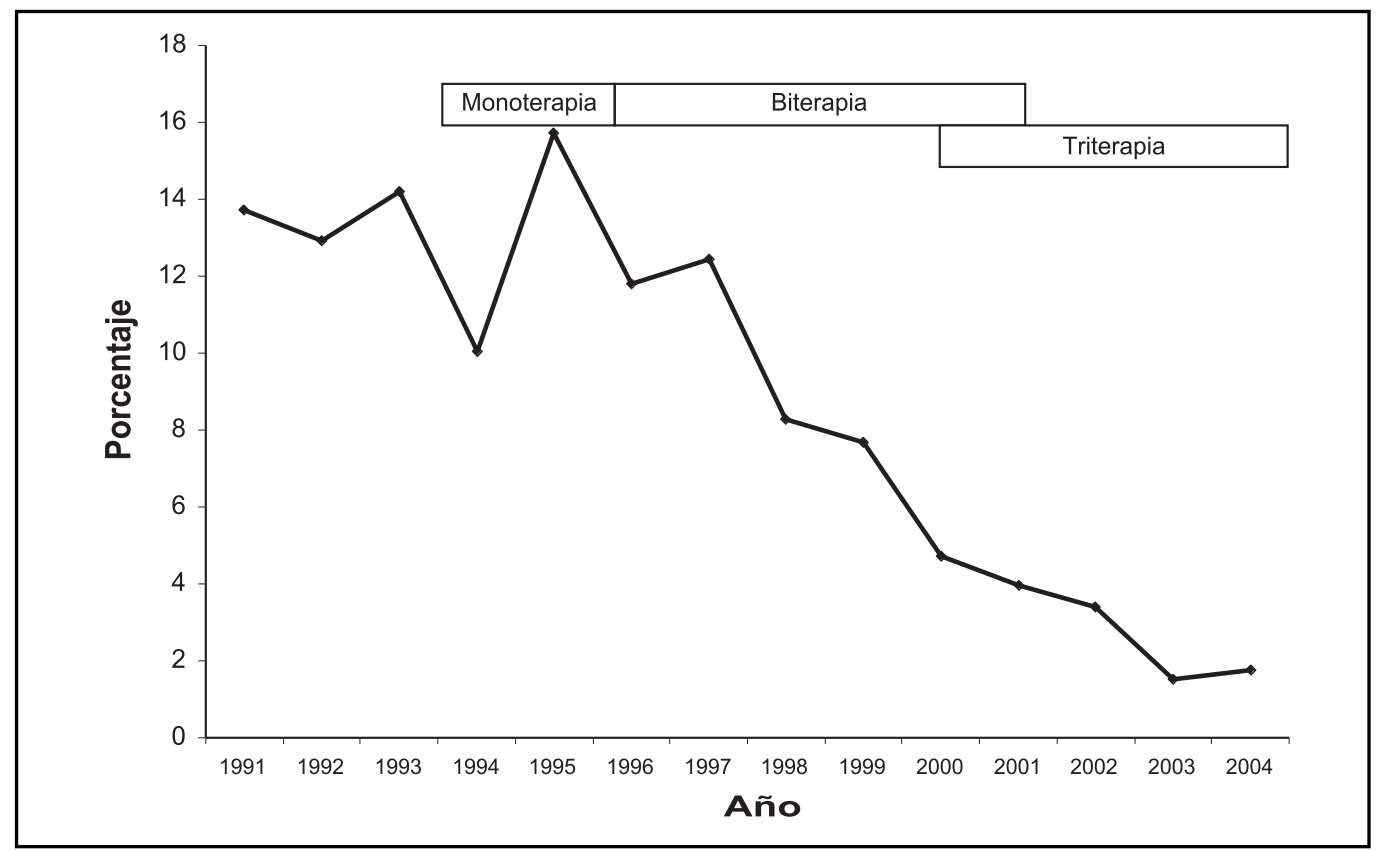

Figura 2. Evolución de mortalidad anual en porcentaje sobre el total de población en seguimiento cada año. Fundación Arriarán, 1991-2004. Monoterapia, biterapia y triterapia= 1, 2 y 3 drogas antirretrovirales, respectivamente.

Tabla 1. Características de la población VIH + atendida en Fundación Arriarán al 31/12/2004

$\begin{array}{ll}\text { Población activa total } & 1.065 \\ \text { Porcentaje hombres } & 87 \% \\ \text { Mediana edad en años } & 36 \\ \text { En terapia antirretroviral } & 817(76,7 \%) \\ \text { En proceso de iniciación o cambio } & 41(4 \%) \\ \text { Sin necesidad o rechazo de terapia } & 207(19,3 \%) \\ \text { Terapia bajo programa de } & \\ \text { acceso expandido } & 758 / 817 \\ \text { Terapia bajo ensayos clínicos } & 59 / 817 \\ \text { Duración terapia (mediana en meses) } & 40\end{array}$

En el último recuento realizado, la mediana había subido a $251,5 \times \mathrm{mm}^{3}$ (aumento absoluto de $96 \%$ ) y la proporción de $<200$ disminuido a $36,8 \%$ (disminución absoluta de 33,4\%), y en el grupo con mayor inmunosupresión, $<100$ a 10,6\% (disminución absoluta de 34,7\%). La mediana de seguimiento fue de 40 meses.

\section{DisCUSIÓN}

Este trabajo tiene por objeto presentar el estado del programa de atención de una población VIH (+) en un centro público multiprofesional. Los resultados son altamente favorables y comparables a centros de países industrializados. Reflejan 
Tabla 2. Regímenes terapéuticos y porcentaje de indetectabilidad viral ( $<400$ copias $x \mathrm{~mm}^{3}$ ) luego de 3 o más meses de terapia* $n=760$

\begin{tabular}{|llrc|}
\hline & Régimen & $\mathrm{n}(\%)$ & \% indetectable \\
\hline 1. & Combivir $^{\circledR}$ y nevirapina & $235(31)$ & 78 \\
2. & Combivir $^{\circledR}$ y efavirenz & $229(30)$ & 80 \\
3. & Lopinavir/ritonavir más otros $_{4}$ & $48(6,3)$ & 58 \\
4. & Didanosina, lamivudina y efavirenz & $44(5,8)$ & 86 \\
5. & Estavudina, lamivudina y nevirapina & $22(2,9)$ & 77 \\
6. & Combivir & e indinavir \\
7. & Didanosina, lamivudina y efavirenz & $18(2,4)$ & 50 \\
8. & Otros & $12(1,6)$ & 44 \\
\hline
\end{tabular}

*Último recuento disponible al 31/12/2004.

Tabla 3. Indetectabilidad viral ( $<400$ copias $x \mathrm{~mm}^{3}$ ) en pacientes con $\geq 3$ meses de terapia antirretroviral continua*

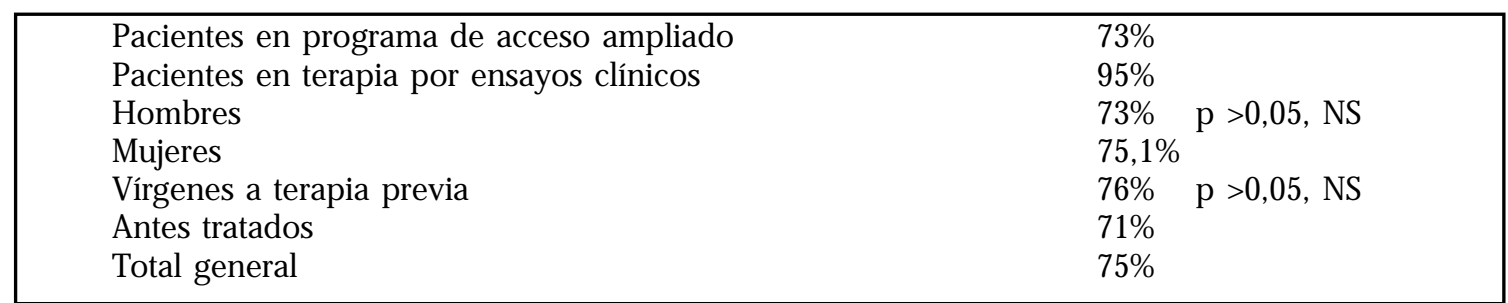

*Último recuento disponible al 31/12/2004.

Tabla 4. Evolución inmunológica durante la terapia antirretroviral CD $4 \times \mathrm{mm}^{3}$

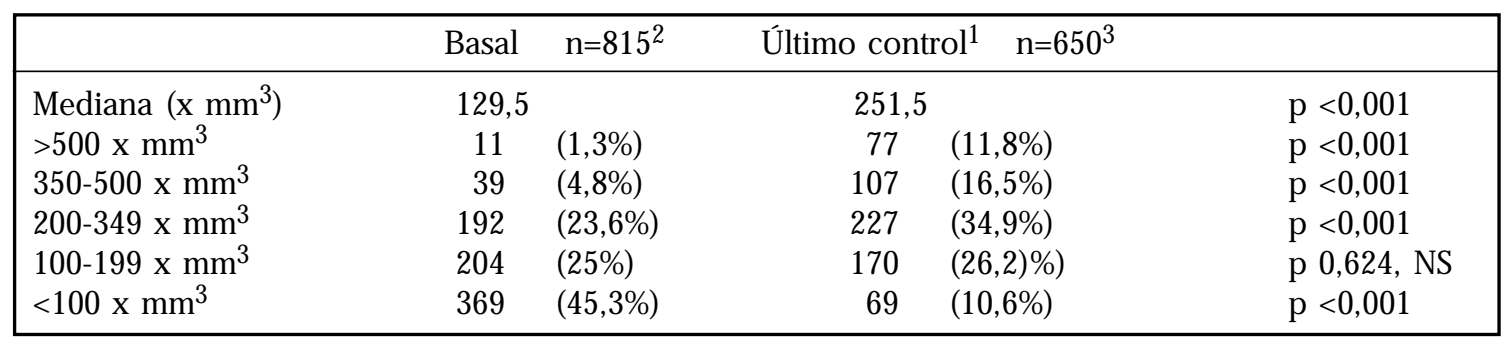

$1=$ al menos 3 meses de terapia antirretroviral y disponible al 31/12/2004. $2=2$ sin registro. $3=113$ sin registro.

lo que está pasando en el resto del país, en donde una red de 32 centros públicos está aplicando el programa de acceso ampliado a TAR en cerca de 5.000 pacientes, con resultados preliminares igualmente favorables ${ }^{7-9}$. La pandemia de SIDA es un modelo sanitario y científico que marcará un hito médico a futuro: en 25 años se ha constituido en el principal problema de salud pública mundial, se prevé que se expandirá masivamente a las áreas más pobladas del globo: China, India y el resto de Asia. En este período se ha podido: caracterizar a cabalidad la enfermedad y su epidemiología, identificar su etiología, implementar técnicas simples de diagnóstico y, más reciente- 
mente, desarrollar medicamentos que transforman la infección, previamente de alta y rápida letalidad, en una condición crónica tratable. Se cuenta con sofisticadas técnicas de monitoreo de esta terapia y se está definiendo su espectro de complicaciones a largo plazo. En el lado negativo, destaca el nulo avance en el desarrollo de una vacuna eficaz, única herramienta de solución global del problema. Se puede decir que el mayor desafío mundial actual no es científico sino logístico: como poner a disposición de los millones de infectados estos adelantos, ya que a nivel mundial menos de $10 \%$ de ellos recibe tratamiento.

Afortunadamente Chile ha podido hacerlo, mayoritariamente, con recursos propios. La experiencia de Fundación Arriarán ha sido relevante. De este centro surgió la primera caracterización clínica y epidemiológica de la enfermedad en el país, hace más de una década ${ }^{10}$. Posteriormente se evaluó el impacto de la terapia en mortalidad ${ }^{11}$ $\mathrm{y}$, luego, la sobrevida y morbilidad en población con y sin acceso a tratamiento ${ }^{6}$, cuyos resultados sin duda apuraron la decisión ministerial de proporcionar terapia moderna de alta efectividad en el país, primero con cobertura incompleta de la población que la requería, para llegar con una expansión creciente al 100\% actual. Se presenta aquí una perspectiva evolutiva de todo este proceso. La reducción de mortalidad anual en, prácticamente, $90 \%$ en tan sólo 9 años, gracias a la implementación de TAR con acceso ampliado a la población que la requería, es indudablemente un resultado exitoso y pocas veces visto con otras enfermedades. Esta reducción se vio luego del inicio de la biterapia, pero también se documentó que este efecto era parcial y transitorio ${ }^{6}$. Mundialmente, la verdadera modificación del pronóstico de la enfermedad se logró con la terapia de alto grado de efectividad (triterapia), hecho también observado en la población de este estudio ${ }^{2,3}$.

En vez de presentar los datos en la forma clásica de sobrevida actuarial, proporción de pacientes con CV indetectable persistente y resultados inmunológicos de acuerdo a regímenes terapéuticos específicos a través del tiempo, este estudio ha preferido una perspectiva epidemiológica práctica: ¿cuál es el estado de una población heterogénea y con regímenes terapéuticos diversos en términos de sobrevida, frenación de la replicación viral y recuperación inmunológica, en un momento dado y luego de un seguimiento a mediano plazo? Esta es la pregunta que este estudio pretende responder. Las limitaciones de un estudio de corte transversal (prevalencia) que sólo registran a los sobrevivientes activos de un seguimiento se ven reducidas en este estudio al incorporar la variación de mortalidad en el tiempo, que en este caso mostró una reducción dramática. La población en tratamiento, luego del plazo requerido para obtener su efecto, presenta un alto nivel de indetectabilidad viral (75\%), comparable a estudios clínicos altamente controla$\operatorname{dos}^{12-14}$ y mejor que los de algunos centros urbanos de países industrializados con población de alta problemática social, en especial drogadicción endovenosa (factor de riesgo de mínima incidencia en la población chilena). Esta importante prevalencia de indetectabilidad se obtiene tanto en población previamente virgen a tratamiento, como aquella con uno o más tratamientos previos y no exitosos, y se da con diversos regímenes terapéuticos, aun cuando este estudio no pretende comparar esquemas de distintos antirretrovirales. Combivir ${ }^{\circledR}$ y efavirenz o nevirapina corresponden en su gran mayoría al primer esquema y de ahí su mayor éxito virológico; los esquemas que incluyen lopinavir/ritonavir son principalmente de rescate frente a fracasos previos, con menores posibilidades terapéuticas de antemano. Un beneficio adicional de una terapia virológicamente efectiva es la marcada reducción en la contagiosidad de los pacientes. Esta situación ya está presente en la mayoría de los pacientes de este centro.

En este estudio no se consignó específicamente el resultado clínico. La forma estándar de clasificar la enfermedad es en asintomáticos (A), con síntomas menores (B) y síntomas mayores (C). La clasificación es unidireccional e irreversible, por lo que una mejoría clínica no se traduce en cambio de etapificación. Sin embargo, dado que las manifestaciones clínicas son tan dependientes del estado inmunológico, se usa la variación del recuento de CD4 para estimar la evolución del estado clínico. Es, por lo tanto, digno de destacar la recuperación inmune de la población tratada. Esta recuperación, un objetivo en sí, está estrechamente ligada a la efectividad de la supresión virológica, pero no siempre requiere de indetectabilidad virológica plasmática, ni tam- 
poco está garantizada por un éxito virológico completo. La recuperación inmune determina el impacto en morbimortalidad. A mayor nivel de CD4, menor morbimortalidad y mejor calidad de vida. El nivel de aumento de CD4 ha sido identificado como una variable independiente de éxito terapéutico en cohortes internacionales ${ }^{15}$. La toxicidad a largo plazo de la TAR está constituyendo un aspecto de cada vez mayor relevancia. Así, ya se están considerando evaluaciones de estrategias terapéuticas guiadas por el nivel de CD4 independiente de la carga viral: tratar sólo si los CD4 están bajos y suspender temporalmente la TAR si suben a un nivel alto predeterminado ${ }^{16}$. El nivel crítico de CD4 para la aparición de complicaciones, necesidad de medicamentos preventivos de éstas y el inicio de TAR es $200 \mathrm{x} \mathrm{mm}^{3}$. La población de este centro ha iniciado TAR con enfermedad avanzada, cerca de $50 \%$ en etapa clínica de SIDA, 70\% con CD4 bajo 200 y 41\% bajo el nivel de 100, nivel que en la cohorte nacional mostró una mortalidad precoz 4 veces mayor que la de 100-200 y 4,6 veces que la de >200 (Cohorte Chilena de SIDA, información no publicada). En este estudio de corte transversal, la población con 3 o más meses de TAR casi había duplicado la mediana de CD4 (129 a 251 x mm³), la proporción con CD4 <200 había caído prácticamente a la $\operatorname{mitad}(70 \%$ a $36 \%)$ y la de mayor riesgo de mortalidad $(<100)$ se había reducido en más de 4 veces $(45,3 \%$ a $10,6 \%)$; todo ello con una muy baja mortalidad en la población estudiada, asegurando que el aumento observado no estaba dado básicamente por fallecimiento de los más inmunodeprimidos y sobrevida de los más inmunocompetentes.

Estos resultados favorables del centro en referencia están siendo reproducidos en la evaluación del impacto del programa a nivel nacional por la

\section{REFERENCIAS}

1. Organización Mundial de la Salud; ONUSIDA: 2004 Informe sobre la epidemia de SIDA, 4o informe mundial; accedido en www.UNAIDS.org, 9 de abril de 2005).

2. Palelia FJ Jr, Delaney KM, Moorman AC, Loveless MO, Fuhrer J, SAtTen GA et al. Declining morbidi-
Cohorte Chilena de SIDA (4.365 pacientes) y, claramente, respaldan la decisión estatal de otorgar tratamiento a la población infectada por VIH. La disminución de los costos de atención de las complicaciones oportunistas ahora evitables, en los gastos de jubilaciones y pensiones prematuras y compensación por pérdida de años de vida productiva debiera permitir financiar el programa. Recientes estudios epidemiológicos en Estados Unidos de Norteamérica (EE.UU) han calculado que las diferentes estrategias de manejo de la epidemia y, en especial la terapia antirretroviral moderna, han salvado más de 2 millones de años de vida en su población; una cantidad igual 0 mayor que la de otras patologías crónicas aún más prevalentes ${ }^{17}$. Es de suponer que resultados proporcionalmente similares se están dando en el país. Estos cálculos no consideran el impacto menos objetivable, pero sí importante, en la aminoración de sufrimiento humano, descomposición familiar y orfandad, así como en la mejoría en autoestima y reinserción laboral y social lograda gracias al tratamiento. La difusión de estos resultados tan exitosos puede ayudar a cambiar la percepción, aún prevalente, de esta enfermedad como rápidamente letal e intratable, no sólo en la población general sino también en muchos profesionales de la salud.

En suma, el programa de acceso ampliado a terapia antirretroviral en un centro especializado en el sector público ha significado una marcada reducción en letalidad de la población en tratamiento, está alcanzando un grado de supresión viral alto y comparable con lo de series internacionales de países industrializados y ha significado una importante recuperación inmune de esta población, todo lo cual se traduce en menor morbimortalidad y mejor calidad de vida.

ty and mortality among patients with advanced human immunodeficiency virus infection. HIV Outpatient Study Investigators. N Engl J Med 1998; 338: 853-60.

3.- Vittinghoff E, Scheer S, O'Mauley P, Colfax G, HOLMBERG SD, BuchBINDER SP. Combination antiretroviral therapy and recent declines in AIDS incidence and mortality. J Infect Dis 1999; 179: 717-20. 
4. Egger M, May M, Chene G, Phimps AN, Lederberger B, Davis F ET AL. Prognosis of HIV-1 infected drug naive patients starting potent antiretroviral therapy: a collaborative analysis of prospective studies. Lancet 2002; 360: 119-29.

5. Wolff M, Beltrán C. Programa de acceso ampliado a terapia antirretroviral en Chile, Plan Auge y Cohorte Chilena de SIDA: Tres iniciativas complementarias. Rev Chil Infectol 2004; 21: 281-4.

6. WolfF M, Diomedi A, Dabanch J, Bidart, Bustamante C, Morales O et al. Seguimiento prospectivo de una población infectada por VIH con y sin posibilidades de terapia antirretroviral: impacto en sobrevida y complicaciones (EFITAR). Rev Méd Chile 2001; 129: 886-94.

7. Vásquez P, Beltrán C, Galardo D, Bustos M, and THE CHILFAN AIDS Study Group. Efficacy of HAART in patients with extremely advanced HIV disease in a middle income country. (Late breaker). $9^{\text {th }}$ European AIDS Conference, Varsovia, Polonia, Octubre 2003 (Disponible en: www.aegis.org/ conferences/ eacs2003).

8. Beltrán C, Wolff MJ, Morales R, Chahin C for the CHILEAN AIDS COHORT. A national cohort model for assessing the effect of EAP for ART and the short term predictors of efficacy in a middle-income country. $11^{\text {th }}$ Conference on Retrovirus and Opportunistic Infections (CROI). Abstract 758. San Francisco, E.U.A, Febrero 2004 (Disponible en: www.retroconference.org/2004/home.htm )

9. Wolff MJ, Beltrán C, Ayala, M, Jenssen W, Hering M, National AIDS Commission (CONASIDA); CHIleAN AIDS Cohort (ChiAC) Study Group. Trends in Antiretroviral (ARV) Drug use in a MiddleIncome Country with an Expanded Access Program (EAP) to HAART. Abstract 890. $42^{\text {nd }}$ Annual Meeting of the Infectious Diseases Society of America (IDSA), Boston, E.U.A., Septiembre 30- Octubre 3, 2004 (Disponible en www.idsociety.org).
10. Wolff M, Northland R, Segovia J, Beltrán C, VaLdÉs M, Albornoz, Auendes G. Características clínicas e historia natural de la infección por Virus de Inmunodeficiencia Humana. Rev Méd Chile 1995; 123: 61-73.

11. Wolff M, Bustamante C, Bidart T, Dabanch J, Diomedi A, Northland R. Impacto de la terapia antirretroviral en la mortalidad de pacientes VIH (+) chilenos: estudio caso-control (MORTAR). Rev Méd Chile 2000; 128: 839-45.

12. Staszewski S, Morales-Ramírez J, Tashima KT, Rachlis A, Skiest D, Stanford J et al. Efavirenz plus zidovudine and lamivudine, efavirenz plus indinavir, and indinavir plus zidovudine and lamivudine in the treatment of HIV-1 infection in adults. Study 006 Team. NEngl J Med 1999; 341: 1865-73.

13. LucAs GM, Chaisson RE, Moore RD. Highly active antiretroviral therapy in a large urban clinic: risk factors for virologic failure and adverse drug reactions. Ann Intern Med 1999; 131: 81-7.

14. Paris D, Ledergerber B, Weber R, Jost J, Flepp M, OPRAVIL $M$ ET AL. Incidence and predictors of virologic failure of antiretroviral triple-drug therapy in a community-based cohort. AIDS Res Hum Retroviruses 1999; 15: 1631-8.

15. Anastos K, Barkón Y, Cohen M H, Greenblatt RM, MinKoff H, Levine A ET AL. The Prognostic Importance of Changes in $\mathrm{CD} 4^{+}$Cell Count and HIV-1 RNA Level in Women after Initiating Highly Active Antiretroviral Therapy. Ann Intern Med 2004; 140: 256-64.

16. ARDuino R. CD4 cell count-guided treatment interuption: be smart and wait for more evidence. Clin Infect Dis 2005; 40: 735-7.

17. Walensky RP, Paltiel A, Losina E, Mercincavage L, Schackman B, Sax P et al. 2 million years of life saved: The survival benefit of AIDS therapy in the United States. Presentado en la $12^{\text {th }}$ Conference on Retrovirus and Opportunistic Infections (CROI). Febrero 22-25, 2005, Boston EEUU (Resumen 143 LB) (Disponible en: www.retroconference.org/ Search_Abstract_2005/AbstractSearch.aspx) 